\section{Revista Facultad 2020 de Ciencias Económicas Vol. 28(1)}

- ISSN: 0121-6805 - ISSN-e: 1909-7719

ISSN:0121-6805 - ISSN

\title{
Derechos humanos, buenas prácticas y responsabilidad social. Aproximación cualitativa en empresas familiares*
}

\author{
Argentina Soto Maciel $^{\mathrm{a}}$ - Odra Angélica Saucedo Delgado ${ }^{\mathrm{b}}$
}

\begin{abstract}
Resumen: El presente documento busca alentar el interés por realizar investigación cualitativa sobre las prácticas laborales en empresas familiares desde la perspectiva de la responsabilidad social empresarial. Para ello, se ponen en perspectiva los derechos humanos laborales fundamentales y su diligencia a través de buenas prácticas laborales, así como la interrelación de estas prácticas con las acciones voluntarias de responsabilidad social empresarial. La interrelación de estos conceptos se aborda desde la perspectiva teórica del modelo bidimensional de la responsabilidad social empresarial, el cual permite discriminar las prácticas laborales en función de la visión y el costo-beneficio que representan para la empresa. El análisis se desarrolla en empresas familiares industriales del sector de la moda en México, seleccionadas a través de una muestra no probabilística y bajo la metodología de caso de estudio. La información recabada y la presentación de resultados es tratada como casos específicos e independientes entre sí. En este sentido, el trabajo que aquí se presenta es de carácter exploratorio y no concluyente; a partir de los casos estudiados se detona la necesidad de replicar este tipo de estudios en muestras amplias para enriquecer el conocimiento sobre el tema.
\end{abstract}

Palabras clave: empresa familiar; derechos humanos laborales fundamentales; buenas prácticas laborales; responsabilidad social empresarial

JEL: M12, M14, M15

Fecha de recepción: 31 de enero de 2020 Fecha de aprobación: 29 de abril de 2020

Disponible en línea: 30 de junio de 2020

Cómo citar: Soto Maciel, A., \& Saucedo Delgado, O. A. (2020). Derechos humanos, buenas prácticas y responsabilidad social. Aproximación cualitativa en empresas familiares. Revista Facultad de Ciencias Económicas, 28(1). https://doi.org/10.18359/rfce.4526

* Artículo de investigación científica y tecnológica. Este trabajo fue posible por la iniciativa y colaboración tripartita de Fondo Semillas, Hispanics In Philanthropy y el Proyecto de Derechos Económicos, Sociales y Culturales. Proyecto: Mejorando las condiciones laborales y de vida para mujeres trabajadoras en México, financiado por la Fundación C\&A.

a Doctora en Ciencias Administrativas, Université Jean Moulin, Lyon 3, Francia. Universidad Anáhuac México, Estado de México, México. Ciudad de México, México. Correo electrónico: argentina.soto@anahuac.mx. ORCID: http://orcid.org/0000-0003-2671-1479

b Doctora en Estudios sobre Desarrollo, Universidad de East Anglia. Universidad Anáhuac México, Estado de México, México. Ciudad de México, México. Correo electrónico: odra.saucedo@anahuac.mx.

ORCID: http://orcid.org/0000-0002-5881-1258 


\title{
Human Rights, Good Practices, and Social Responsibility. Qualitative Approach to Family Businesses
}

\begin{abstract}
This paper seeks to awaken interest in conducting qualitative research into work practices of family businesses from corporate social responsibility. For this, it puts into perspective fundamental labor human rights and their exercise through good labor practices, as well as the interrelation of these practices with voluntary actions of corporate social responsibility. The interrelation of these concepts is theoretically approached from the two-dimensional model of corporate social responsibility, which allows disaggregating labor practices based on the company's vision and cost-benefit. The analysis is carried out on family-owned industrial companies in the fashion industry in Mexico using non-probability sampling and the case study method. The information collected and the results presented are treated as specific and independent cases. Thus, this research is exploratory and inconclusive and the cases studied create the need to replicate this type of study in larger samples to extend knowledge on the subject.
\end{abstract}

Keywords: family business; fundamental labor human rights; good work practices; corporate social responsibility

\section{Direitos humanos, boas práticas e responsabilidade social. Aproximação qualitativa em empresas familiares}

\begin{abstract}
Resumo: Este documento pretende incentivar o interesse em realizar pesquisas qualitativas sobre as práticas trabalhistas em empresas familiares do ponto de vista da responsabilidade social empresarial. Para isso, são colocados em perspectiva os direitos humanos trabalhistas fundamentais e sua diligência, por meio de boas práticas trabalhistas, bem como a inter-relação dessas práticas com as ações voluntárias de responsabilidade social empresarial. A inter-relação desses conceitos é abordada teoricamente a partir do modelo bidimensional da responsabilidade social empresarial, o que permite distinguir as práticas laborais em função da visão e do custo-benefício que representam para a empresa. A análise é desenvolvida em empresas familiares industriais do setor da moda no México, selecionadas por uma amostra não probabilística e com base na metodologia de caso de estudo. A informação coletada e a apresentação de resultados são tratadas como casos específicos e independentes entre si. Nesse sentido, este trabalho é de caráter exploratório e não conclusivo. A partir dos casos estudados, denota-se a necessidade de reproduzir esse tipo de estudos em amostras mais amplas para enriquecer o conhecimento sobre a temática.
\end{abstract}

Palavras-chave: empresa familiar; direitos humanos trabalhistas fundamentais; boas práticas trabalhistas; responsabilidade social empresarial 


\section{Introducción}

La empresa familiar es el principal motor de las economías en todo el mundo por su alta contribución al producto interno bruto y a la generación de empleo (Anderson y Reeb, 2003; Astrachan y Shanker, 2003; Lagos, Betancourt y Gómez, 2018). En la empresa familiar, las interrelaciones entre sus subsistemas, propiedad, empresa y familia (Gersick, Davis, Hampton y Lansberg, 1997), provocan comportamientos y gestión diferenciados. Entre ellos, las empresas familiares generan lazos estrechos con su entorno, por ejemplo, la comunidad, los clientes o los empleados (Uhlaner, Van GoorBalk y Masurel, 2004; Bingham, Dyer, Smith y Adams, 2011; Neubaum, Dibrell y Craig, 2012).

En este contexto, el objetivo del trabajo es realizar una propuesta de análisis y abrir la discusión entre académicos para mejorar la comprensión de la relación que establecen las empresas familiares con sus empleados, tomando en consideración el grado de autonomía con el que conciben y diseñan las prácticas laborales. Proponemos clasificar el grado de autonomía en tres niveles: los derechos humanos laborales fundamentales, las buenas prácticas laborales y la responsabilidad social empresarial. En México, los derechos humanos laborales fundamentales (DHLF) son de carácter obligatorio. Las buenas prácticas laborales (BPL) también presentan este carácter obligatorio, aunque resaltan el aspecto de la predisposición de las empresas para entablar buenas relaciones laborales con sus empleados. Las prácticas de responsabilidad social empresarial (RSE), por su parte, dan evidencia del involucramiento empresarial voluntario sobre preocupaciones sociales y económicas de los trabajadores. Aquí, vale la pena destacar que la relación -y delimitación- entre las BPL y las acciones voluntarias de RSE, particularmente en la dimensión laboral, no han sido suficiente exploradas desde el ámbito de la academia. Igualmente, en el caso del estudio de BPL en la empresa familiar (EF), se carece de registro alguno, lo que incrementa el interés por este tema de investigación.

En ese sentido, una de las principales aportaciones del estudio es su énfasis en el tratamiento de las BPL-de carácter obligatorio-', y su interrelación con el ámbito de voluntariedad de la RSE, a partir de la cual las empresas llevan a cabo acciones concretas. Todo lo anterior viene delimitado por el marco de los DHLF de los trabajadores del sector mexicano de la moda.

En términos conceptuales, las contribuciones de esta investigación se sustentan en el modelo bidimensional de Quazi y O'Brien (2000), el cual permite identificar qué tipo de prácticas laborales lleva a cabo la empresa, establecer su relación con una visión de corto o largo plazo y relacionarla como con una lógica de costo-beneficio.

Este artículo ha sido estructurado en cinco grandes apartados. En el primero, se describen los DHLF vigentes en México, sus implicaciones para las prácticas laborales, así como la distinción conceptual entre BPL y acciones de RSE. En el segundo apartado, se presenta el modelo bidimensional de la RSE, a través del cual se analizan las prácticas laborales según la visión en el tiempo y la lógica costo-beneficio que representan para la empresa. En el tercero, se define el concepto de Empresa Familia (EF), relevante para este trabajo, y se refuerza la pertinencia de considerarlo en este estudio. En la cuarta sección, se presenta la metodología de tipo exploratorio y cualitativo, basada en tres casos de estudios independientes, para dar paso al análisis y la presentación de resultados. En la última sección se presentan las conclusiones, limitaciones y futuras líneas de esta investigación.

\section{Derechos humanos laborales fundamentales (DHLF), buenas prácticas laborales (BPL) y responsabilidad social empresarial (RSE)}

Inicialmente, es relevante destacar que México ha sido el primer país en reconocer los derechos laborales, lo cual se encuentra plasmado desde la Constitución de 1917 (Comisión Nacional de los Derechos Humanos [CNDH], 2016; Diario Oficial de la Federación [DOF], 1917). Adicionalmente, existen diversos instrumentos internacionales que reconocen el derecho al trabajo y los derechos humanos en el trabajo, los cuales han sido suscritos 
por este país. Entre ellos se destacan "La Observación General n. ${ }^{\circ}$ 18, El derecho al Trabajo" (Comité de Derechos Económicos, Sociales y Culturales, 2005) y los ocho convenios fundamentales emitidos por la Organización Internacional del Trabajo (OIT, 1996-2020) ${ }^{1}$.

En México, los derechos humanos fundamentales están considerados dentro del marco legal vigente y están previstos en los artículos $5^{\circ}$ y 123 de la Constitución Política de los Estados Unidos Mexicanos (DOF, 1917), así como en sus leyes reglamentarias, Ley Federal del Trabajo y Ley Federal de los Trabajadores al Servicio del Estado (CNDH, 2016; DOF, 1970; DOF, 1963).

En el siguiente apartado se describen los DHLF vigentes en el país, así como sus implicaciones dentro del ámbito laboral.

\section{DHLF en México}

De acuerdo con la legislación mexicana, los derechos humanos laborales fundamentales ${ }^{2}$ son la base para el mejoramiento de las condiciones de vida del trabajador en la empresa, al garantizar su libertad e igualdad. Estos se desglosan en diez derechos (Tabla 1).
Tabla 1. DHLF y sus implicaciones para las prácticas laborales

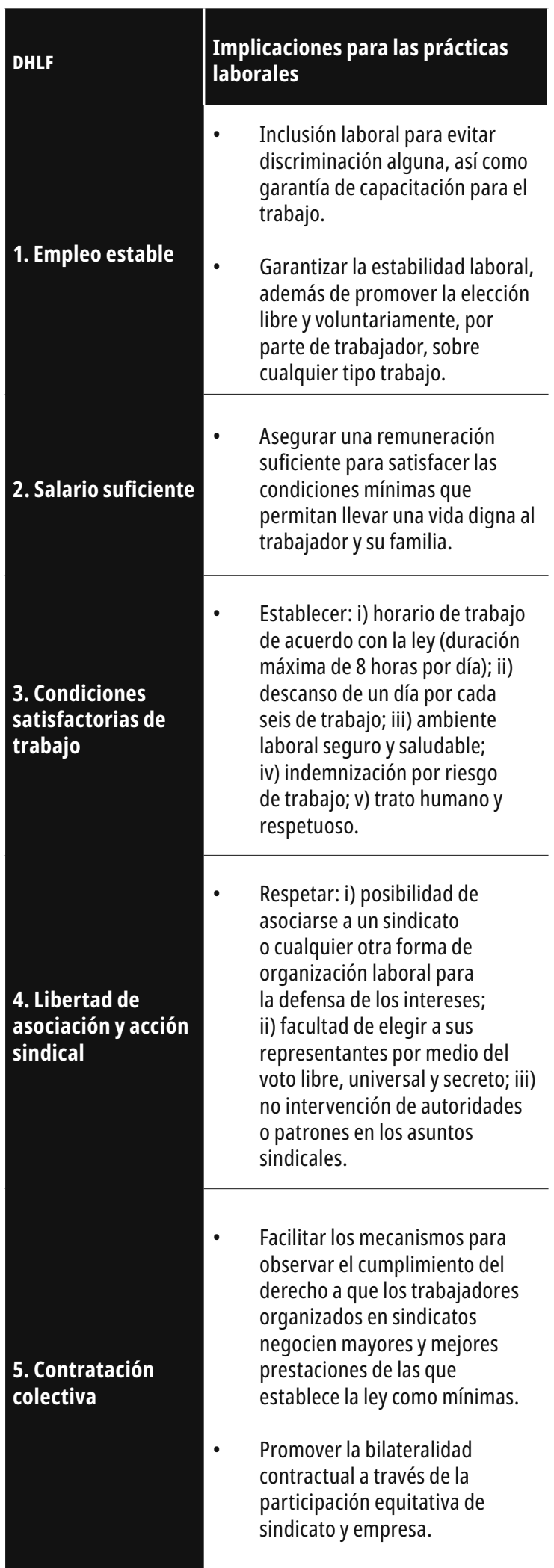




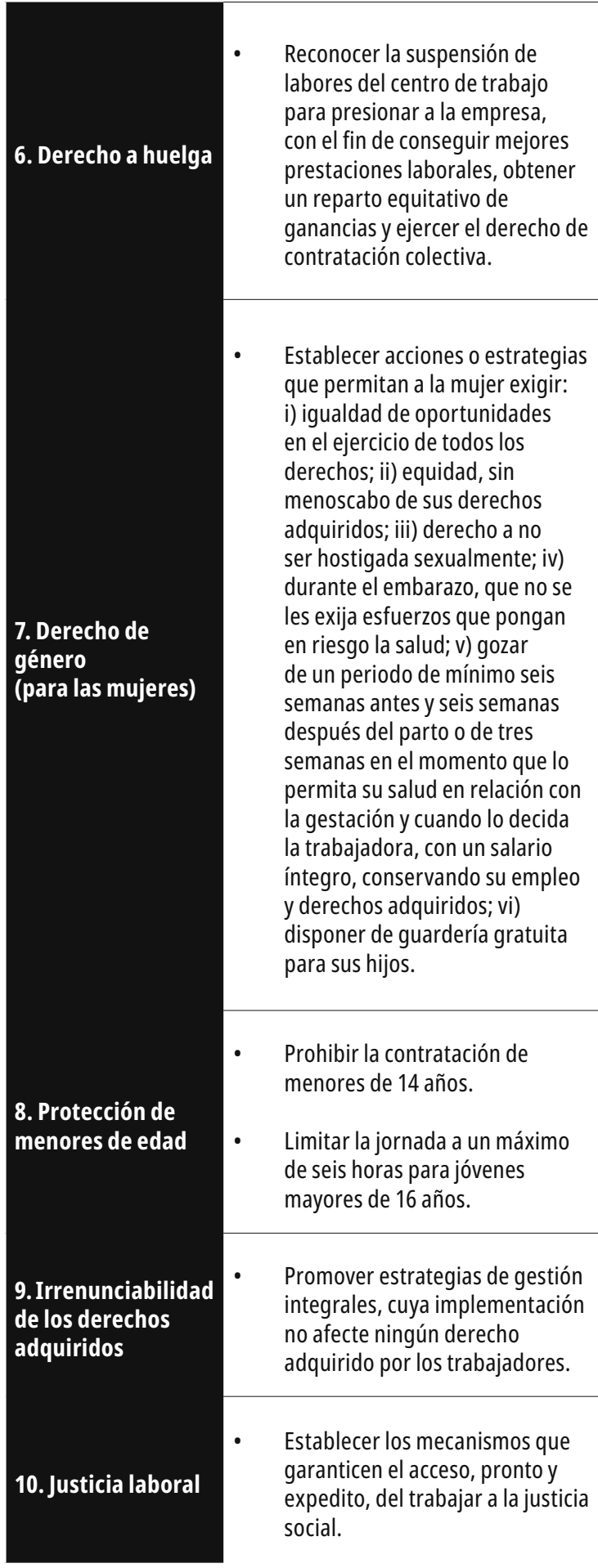

Fuente: elaboración propia con datos de la Organización Internacional del Trabajo (oit, 2009; 2018).

Estos DHLF son integrales, indivisibles e interdependientes (ACNUDH, 1996-2019). Y dado su carácter obligatorio, en ellos se sustentan las BPL.

\section{Buenas prácticas laborales (BPL)}

De manera extendida, el término de BPL suele referirse a las experiencias exitosas que responden a una necesidad creciente por buscar "mecanismos o formas de interacción" que den acceso a los trabajadores y trabajadoras al ejercicio pleno de sus derechos laborales fundamentales (Rueda-Catry y Ruiz, 2005). De ahí que su observancia sea de carácter obligatorio.

Aunque el concepto aún está en construcción, instituciones como la Organización Internacional del Trabajo han trabajado buscando su consolidación. De acuerdo con la recomendación 195 (OIT, 2005), los empleadores -tanto públicos como privados- deben implementar BPL en materia de desarrollo de los recursos humanos. Estas iniciativas deben considerar por lo menos tres perspectivas: la jurídica, la de clima laboral y la de beneficio a los trabajadores.

No obstante, Eróstegui (2011) considera que existen dos circunstancias esenciales para la implementación de BPL. La primera de ellas es que este tipo de prácticas no deben limitarse al carácter obligatorio de la normatividad laboral vigente y la segunda, que estas deben estar orientadas a cuidar integralmente a los empleados. De ahí que tienda a traslaparse este término con las acciones voluntarias de RSE y se torne difícil su disociación.

\section{Prácticas laborales de RSE}

Como se mencionó con anterioridad, las prácticas de RSE son voluntarias y de tendencia innovadora (Reyno, 2006). Algunos factores, como cambios en la industria, en las regulaciones o en los valores sociales, aceleran significativamente la integración de ellas dentro de las empresas (Cairncross, 1992; Judge y Douglas, 1998; Sharma y Srinivas, 2000).

Por lo previamente expuesto en este trabajo, la distinción entre normatividad vigente inscrita en los DHLF, las BPL y las acciones voluntarias de RSE es fundamental para que las BPL no se desarticulen del ámbito de las obligaciones legales bajo la apariencia de pertenecer al ámbito voluntario de la RSE. Adicionalmente, las prácticas documentadas en el estudio deben hacer énfasis en las condiciones laborales de las trabajadoras, pues el grupo 
poblacional de las mujeres tiene una representación considerable en el sector de la moda mexicano, el cual incluye empresas orientadas a la fabricación de ropa, calzado y diversos artículos en piel u otros materiales, también accesorios, conocidos como bisutería. Dicho sector se inserta dentro de la industria textil y de la confección. Según cifras de Inegi (2020), alrededor del 60\% del personal ocupado en este sector son del género femenino.

\section{Modelo bidimensional de la RSE}

El modelo bidimensional de RsE (Quazi y O’Brien, 2000) clasifica las prácticas de acuerdo con la visión empresarial y la razón costo-beneficio para la empresa (ver Figura 1).

El modelo está compuesto por dos ejes. El eje horizontal representa la visión de las prácticas de RSE, que puede ser reducida o amplia. En la primera, la prestación de bienes o servicios tiene una lógica de maximización de utilidades en el corto plazo y se limita a la regulación del contexto donde se ubica la empresa. En la segunda, la empresa tiene vocación de servir a la sociedad en la protección ambiental, el desarrollo de la comunidad, la conservación de recursos o la filantropía. El eje vertical representa la razón costo-beneficio para la empresa. El extremo inferior y negativo representa el costo de la acción en el corto plazo. El extremo superior y positivo, el costo en el largo plazo.
Enseguida, emanan cuatro perspectivas: i) clásica; ii) socioeconómica; iii) filantrópica; y iv) moderna. En la perspectiva clásica, la finalidad de la empresa es la maximización de utilidades, por lo que las acciones de RSE solo le generan costo. En la socioeconómica, la visión de la empresa hacia la RSE es estrecha, sin embargo, acepta que algunas acciones pueden generarle beneficios, como establecer buenas relaciones con proveedores y consumidores. En la perspectiva filantrópica, la empresa acepta participar en actividades caritativas o humanitarias, pero aún son percibidas como un costo. Su participación solo responde a su relación altruista o ética con la sociedad. Finalmente, en la perspectiva moderna, la empresa logra beneficios de sus acciones de RSE en el corto y largo plazo, en especial con sus grupos de interés.

En este trabajo, las prácticas laborales inscritas en los DHLF representan la perspectiva clásica; las bpl, la socioeconómica; las prácticas de rse, la moderna; mientras que las prácticas humanitarias, la filantrópica.

\section{La empresa familiar}

El concepto de empresa familiar cuenta con una amplia variedad de acepciones y numerosos trabajos dan cuenta de ello (Benavides, Guzmán y Quintana, 2011; Yu, Lumpkin, Sorenson y Brigham, 2012; Harms, 2014; Molina, Botero y

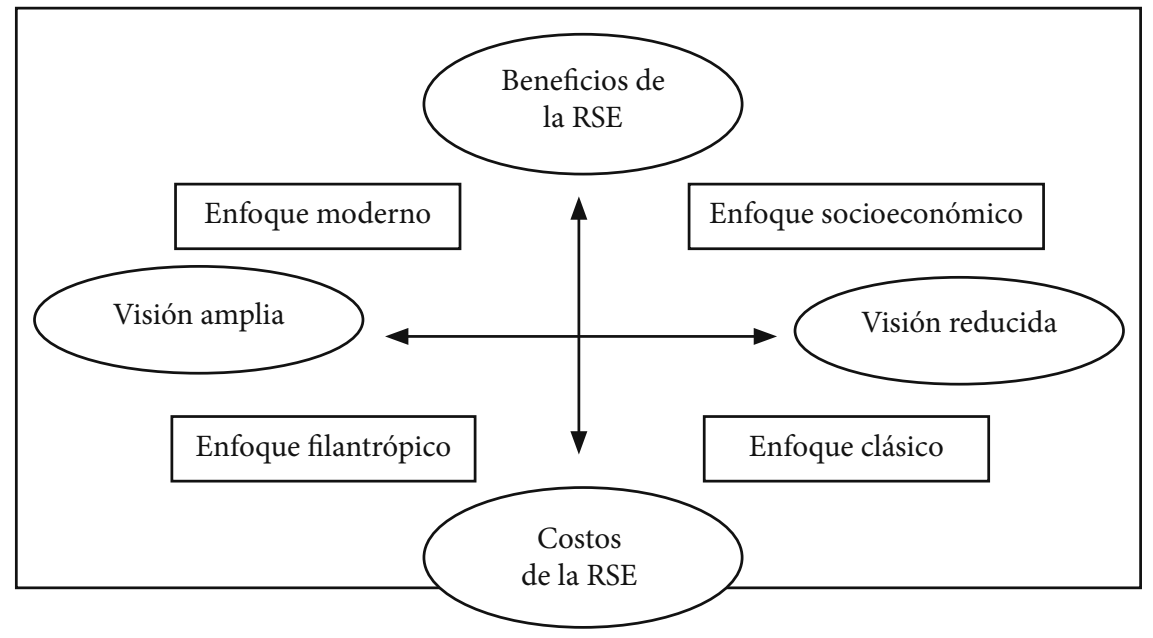

Figura 1. Modelo bidimensional de RSE.

Fuente: Quazi y O'Brien (2000, p. 35). 
Montoya, 2016). Aunque se carece de acuerdo, en la empresa familiar se destacan algunas condiciones, como el hecho de que los miembros familiares sean propietarios de la empresa (Dyer, 1986), participen dentro en la dirección o la toma de decisiones (Handler, 1989) o que ambas condiciones sean reunidas (Sharma et al., 1997; Cromie, Dunn, Sproull y Chalmers, 2001; Sharma, 2004). En consecuencia, las interrelaciones que se producen entre la familia y el sistema familiar son lo que genera en ellas un comportamiento único (Gersick et al., 1997). En este trabajo, se considera como EF aquella donde varios miembros familiares participan tanto en la propiedad, como en su dirección.

El interés por estudiar la RSE en la ef surge en 1990. Los primeros trabajos comparan los comportamientos entre empresas familiares y no familiares (Van Gils, Dibrell, Neubaum y Craig, 2014). Inicialmente, los resultados evidencian que los comportamientos no son solamente diferenciados, sino que existe mayor propensión hacia prácticas de rse en las empresas familiares (Adams, Taschian y Shore, 1996; Graafland, 2002; Gallo, 2004; Dyer y Whetten, 2006; Campopiano y De Massis, 2015; Castejón y López, 2016; Hernández-Calzada et al., 2017). Por ejemplo, en contraste con las empresas no familiares (enf), las ef son más propensas a: promover comportamientos éticos por considerarlos más importantes (Adams et al., 1996); repartir riqueza y entregar productos para el bien de la comunidad (Gallo, 2004); manifestar compromiso, apoyo y sentido de comunidad, donde su tamaño se relaciona con la capacidad para dar un beneficio comunitario (Niehm, Swinney y Miller, 2008); comprometerse con las preocupaciones sociales (Dyer y Whetten, 2006) y ambientales (Huang, Ding y Kao, 2009); difundir una mayor variedad de reportes de RSE y mostrar mayor interés con diferentes temas al respecto (Campopiano y De Massis, 2015).

Otros trabajos se enfocan en estudiar a la EF y su relación con la RSE. En ellos, se reconoce que estas empresas conservan relaciones personales, contacto directo y consideración con empleados (Gil, Jiménez y Lorente, 2001; Huang et al., 2009), o con empleados y clientes (Flören, Leach y Wijers, 1993; Uhlaner et al., 2004; Bingham et al., 2011;
Neubaum et al., 2012); y que aquellas que tienen prácticas de RSE son más atractivas para los trabajadores potenciales.

Sin embargo, otros trabajos cuestionan esa relación positiva (Cabrera et al., 2005; Déniz y Cabrera, 2005; Suárez, Déniz y Santana, 2011). Por ejemplo, los resultados de Cuadrado-Ballesteros et al. (2015) muestran que en las EF se antepone el interés financiero a las prácticas de RSE; o que la influencia familiar reduce su compromiso hacia la RsE (Cabeza-García et al., 2017), por lo que se estima que el comportamiento de la EF ante la RSE no es inherente a su naturaleza, sino contingente (Niehm et al., 2008; Uhlaner et al., 2004; Sharma, 2004), donde algunos factores que se destacan son la tradición o los valores familiares (Marques, Presas y Simon, 2014); el tamaño (Amato y Amato, 2007; Perrini, Russo y Tencati, 2007; Nisim y Benjamin, 2008; Amann, Jaussaud y Martínez, 2012; Gavana, Gottardo y Moisello, 2017); la innovación (Amann et al., 2012); el liderazgo generacional, el nivel de control o la participación familiares en la toma de decisiones (Sharma, 2004; Déniz y Cabrera, 2005; Quintana, 2005; Campopiano, De Massis y Cassia, 2012). Para Uhlaner et al. (2004) y Niehm et al. (2008) los factores determinantes dependen del tamaño, el sector y la generación, mientras que para Suárez et al. (2011) los factores que favorecen la institucionalización de prácticas de RSE pueden agruparse en sectoriales, organizativos y de entorno.

\section{Metodología}

Gummesson (2015) afirma que la metodología cualitativa y los casos de estudio proveen, al investigador sobre empresas, de herramientas relevantes para llevar a cabo su trabajo. A través de ellas, la aplicación, el énfasis y el significado varían considerablemente por relacionarse con la vida humana en diferentes escenarios, persiguiendo diferentes propósitos y desde distintas perspectivas.

El estudio de caso es un enfoque prominente en la investigación de la EF de corte cualitativo (Leppäaho, Plakoyiannaki y Dimitratos, 2016). Esta herramienta puede definirse como el estudio de la particularidad y complejidad de uno o más casos con el fin de comprender su actividad dentro de 
ciertas circunstancias importantes (Stake, 1995), al abordar preguntas sobre "cómo" o "por qué" de un fenómeno y de su contexto (Yin, 2017). De Massis y Kotlar (2014) confirman su pertinencia al afirmar que los negocios familiares son un fenómeno diferente que debe ser estudiado desde diversos enfoques teóricos y niveles de análisis, mientras que Yin (2017) reconoce su capacidad para investigar un fenómeno contemporáneo en el contexto de la vida real. Al respecto, el presente trabajo se desarrolla en empresas familiares industriales del sector de la moda en México, seleccionadas a través de una muestra no probabilística (o por conveniencia), donde las personas entrevistadas fueron elegidas con base en criterios de accesibilidad y facilidad operativa.

La información recabada fue tratada como estudios de caso específicos e independientes entre sí. En este sentido, el trabajo que aquí se presenta es de carácter exploratorio y no concluyente $(\mathrm{Mu}-$ ñoz, 2011). Es decir, su escala es pequeña y permite hacer algunas aproximaciones empíricas sobre el tratamiento de las buenas prácticas laborales y acciones de RSE de tres empresas en el marco de los derechos humanos laborales fundamentales de los trabajadores del sector mexicano de la moda.

Si bien el propio diseño metodológico de este estudio tiene como consecuencia la imposibilidad de hacer generalizaciones y afirmaciones directas con respecto a las empresas participantes -o al resto de empresas que operan dentro del sector moda en México-, una de las ventajas de la investigación exploratoria radica en su flexibilidad de diseño. Esto permite adaptarse al tamaño de la muestra, pero también estar abierta a nuevas soluciones, u orientaciones, conforme se amplía el número de datos o casos de estudio (Ghauri y Grønhaug, 2020, pp. 63-64).

Asimismo, esta investigación ha optado por un diseño exploratorio debido a la falta de acceso a datos de campo para emprender un análisis más profundo. Sin embargo, una de sus principales contribuciones radica en que aumenta el grado de familiaridad con un tema poco explorado como es el de la interrelación de los conceptos de DHLF, BPL y RSE. Con ello se abre la posibilidad de establecer prioridades en la agenda para llevar a cabo una investigación más completa sobre un contexto determinado como es la empresa familiar en México, en particular dentro de la industria de la moda (Fletcher, De Massis y Nordqvist, 2015).

La recolección de los datos se obtuvo a través de entrevistas enfocadas y semiestructuradas. En total se realizaron tres entrevistas a los responsables del área de Recursos Humanos. Si bien el número de participantes es reducido, en estudios cualitativos el tamaño de la muestra está estrechamente vinculado al diseño metodológico y proceso de saturación de la información que no responde a un proceso de aplicación estandarizada (Malterud, Volkert y Guassora, 2015). En este sentido, en la selección de los casos predominó el criterio de exploración profunda, más que el de representatividad. Este último se basa en un número más extenso de personas entrevistadas. Por ello se dio prioridad a una recolección de datos basada en el objetivo del estudio y la calidad del diálogo con los entrevistados, así como en la estrategia de análisis temático de los datos.

A partir de lo anterior, las entrevistas se llevaron cabo por videoconferencia, lo que facilitó su grabación para posterior transcripción estenográfica. Cada uno recibió con antelación una invitación para participar y la guía de temas a tratar durante la conversación. La colaboración fue voluntaria y la información proporcionada manejada cuidadosamente. La información recabada para cada caso corresponde a la experiencia y entorno específico de cada empresa, por lo que no todos los temas fueron abordados en el mismo orden o nivel de detalle.

Por su parte, como ya se ha mencionado, el tratamiento de datos se condujo a través de un análisis temático. Esta técnica es ampliamente utilizada dentro de los estudios cualitativos. Su flexibilidad permite extraer significados y conceptos que permiten establecer patrones de temas recurrentes en los datos obtenidos a través de las entrevistas realizadas (Javadi y Zarea, 2016; King y Brooks, 2018).

Aunado a ello, con el objetivo de contrarrestar el carácter libre, discrecional o prudencial de 
Tabla 2 .Características demográficas

\begin{tabular}{lllll} 
& Caso 1 & Caso 2 & Caso 3 \\
\cline { 2 - 4 } Actividad & Fabricación de calzado. & $\begin{array}{l}\text { Fabricación de bolsos, calzado, } \\
\text { accesorios y equipaje. }\end{array}$ & $\begin{array}{l}\text { Fabricación de camisas y ropa } \\
\text { de trabajo pesado. }\end{array}$ \\
\hline Año de creación & 1978 & 1989 & 1940 \\
\hline Número de empleados & 540 & 622 & 370 \\
\hline Características laborales & $43 \%$ mujeres. & $64 \%$ mujeres. & $60 \%$ mujeres. \\
\hline Liderazgo familiar & 2da generación, & género masculino. & $\begin{array}{l}\text { 2da generación, } \\
\text { género masculino. }\end{array}$ & 3era generación, \\
& & & género masculino.
\end{tabular}

Fuente: elaboración propia con datos de Hispanic in Philantropy [hip] (2018).

las prácticas laborales, conservamos solo aquellas que son sistemáticas de carácter universal, accesibles a todos los empleados. Los resultados de esta recolección de datos fueron analizados y sintetizados para presentarse de forma esquemática, como se observa en la siguiente sección.

\section{Aproximación a las prácticas laborales en empresas familiares}

Las EF participantes en el estudio son industriales y de tamaño grande de acuerdo con el número de

Tabla 3. Certificaciones y reconocimientos

\begin{tabular}{|c|c|c|c|}
\hline & Caso 1 & & Caso 3 \\
\hline Empresariales & $\begin{array}{l}\text { Calidad (IS0 9000) } \\
\text { Ambiental } \\
\text { (Iso 14000) } \\
\text { Comercialización internacional (Premio } \\
\text { Nacional de Exportación) } \\
\text { Recursos humanos (Reconocimiento } \\
\text { Estatal al Mérito Laboral) }\end{array}$ & & \\
\hline rse & & $\begin{array}{l}\text { ESR }\left(\text { Cemefi }{ }^{3} \text { ) }\right. \\
\text { (Por el apoyo a Fundaciones: de } \\
\text { los enfermos en pobreza; contra } \\
\text { el cáncer de mama; contra la } \\
\text { anorexia y bulimia - padecimientos } \\
\text { asociados al mundo de la moda) }\end{array}$ & $\begin{array}{l}\text { Empresa incluyente }\left(\text { STPS }^{4} \text { ) }\right. \\
\text { Certificado Internacional } \\
\text { WRAP }^{5}\end{array}$ \\
\hline
\end{tabular}

Fuente: elaboración propia con datos de hip (2018).

3 Centro Mexicano para la Filantropía.

4 Secretaría del Trabajo y Previsión Social.

5 Word Responsible Accredited Production. 
Tabla 4. Síntesis de las prácticas laborales de empresas familiares de la moda

\begin{tabular}{|c|c|c|c|}
\hline & Caso 1 & Caso 2 & Caso 3 \\
\hline \multicolumn{4}{|l|}{ Perspectiva clásica } \\
\hline Acceso y garantía de estabilidad & Sí & Sí & Sí \\
\hline Inclusión y equidad de género & Sí & Sí & Sí \\
\hline Condiciones de trabajo & Sí & Sí & Sí \\
\hline Prevención y atención del acoso sexual & Sí & Sí & Sí \\
\hline \multicolumn{4}{|l|}{ Perspectiva socioeconómica } \\
\hline Salud y bienestar físico & $\begin{array}{l}\text { Prevención de } \\
\text { padecimientos } \\
\text { asociados a la actividad } \\
\text { laboral. }\end{array}$ & $\begin{array}{l}\text { - Prevención de riesgos } \\
\text { asociados a la actividad } \\
\text { laboral. }\end{array}$ & $\begin{array}{l}\text { - Prevención de } \\
\text { padecimientos y riesgos } \\
\text { asociados a la actividad } \\
\text { laboral. }\end{array}$ \\
\hline Conciliación trabajo-familia & $\begin{array}{l}\text { - Actividades } \\
\text { recreativas. }\end{array}$ & $\begin{array}{l}\text { Actividades sociales. } \\
\text { - Flexibilidad en los } \\
\text { permisos por motivos } \\
\text { familiares. }\end{array}$ & - Actividades deportivas. \\
\hline $\begin{array}{l}\text { Acciones de apoyo a la salud } \\
\text { reproductiva }\end{array}$ & $\begin{array}{l}\text { § Seguimiento médico. } \\
\text { § Asignación de } \\
\text { actividades sin riesgo. }\end{array}$ & $\begin{array}{l}\text { § Seguimiento médico. } \\
\text { § Flexibilidad en los } \\
\text { permisos. } \\
\text { § Disposición de un } \\
\text { espacio de lactancia. }\end{array}$ & $\begin{array}{l}\text { Seguimiento médico. } \\
\text { - Asignación de actividad } \\
\text { sin riesgo. } \\
\text { - Flexibilidad de horarios. } \\
\text { - Disposición de un } \\
\text { espacio de lactancia. }\end{array}$ \\
\hline \multicolumn{4}{|l|}{ Perspectiva filantrópica } \\
\hline $\begin{array}{l}\text { Iniciativas de rse, orientadas al } \\
\text { desarrollo profesional y humano de los } \\
\text { trabajadores }\end{array}$ & $\begin{array}{l}\text {. Pláticas y estrategias } \\
\text { orientadas al } \\
\text { crecimiento personal. }\end{array}$ & $\mathrm{N} / \mathrm{A}$ a/ & - Alfabetización \\
\hline \multicolumn{4}{|l|}{ Perspectiva moderna } \\
\hline Prácticas de rse & - Código de Ética. & . Código de Conducta. & . Código de Conducta. \\
\hline
\end{tabular}

a/Dentro de las acciones filantrópicas de la empresa, se destacan su participación en las campañas anuales para la atención del cáncer de mama y trastornos de anorexia y bulimia (hip, 2018: 15).

Fuente: elaboración propia con datos de hip (2018).

empleados ${ }^{6}$ (en promedio, cuentan con 510 empleados, ver Tabla 2). La participación del género femenino representa en promedio $56 \%$ del total de la planta laboral y se concentra en el área de producción. La antigüedad promedio de las empresas es de 49 años (oscilan entre 30 y 78 años de existencia). En todas ellas, se destaca el liderazgo familiar, masculino, de segunda y tercera generación, así como la participación de ambas

6 La empresa industrial es grande a partir de 251 empleados (DOF, 2009), consultado en www.dof.gob.mx generaciones en la dirección general o en la presidencia del consejo. Los fundadores, inclusive retirados, frecuentan con regularidad la empresa.

En su origen, las empresas tenían una capacidad de producción artesanal o industrial de muy pequeña escala, cuya producción se destinaba al mercado local o nacional. En la actualidad, las empresas cuentan con una importante capacidad productiva y su mercado es internacional. A pesar de esa exposición, la importancia de las certificaciones es heterogénea (ver Tabla 3). 
Entre las certificaciones se destaca la importancia de las auditorías externas de clientes internacionales. Lo que demuestra una mayor presión comercial que operativa en las empresas.

Las prácticas laborales identificadas se sintetizan en la siguiente tabla.

a/Dentro de las acciones filantrópicas de la empresa, se destacan su participación en las campañas anuales para la atención del cáncer de mama y trastornos de anorexia y bulimia (HIP, 2018: 15). Fuente: elaboración propia con datos de HIP (2018).

Las empresas participantes se encuentran alineadas al marco legal vigente en México, es decir, la Ley Federal del Trabajo (DOF, 1970), que es de observancia general en todo el país y rige las relaciones laborales en función del artículo 123 constitucional. Es importante destacar que este artículo es pilar fundamental para la protección y ejercicio pleno de los DHLF, dentro del ámbito nacional (CNDH, 2016). En consecuencia, la perspectiva clásica reúne los cuatro DHLF previstos: empleo estable, salario suficiente, condiciones satisfactorias de trabajo y derecho de género. Sin embargo, están ausentes los derechos laborales referentes a la libertad de asociación y acción sindical, ya que resultó un tema sensible para las empresas, por lo que se prefirió omitir de las entrevistas. En ellas se establecen relaciones contractuales indefinidas o temporales, salario de base, incentivos, prestaciones (seguridad social y fondo para la vivienda), atención médica, igualdad de acceso al trabajo y al salario, equidad de género, así como prevención y atención del acoso e inclusión.

Sobre la equidad de género, las entrevistas manifiestan que es aplicable a todas las áreas de la empresa. Sin embargo, al contrastar dicha afirmación con la información recolectada, se identifica que los puestos de mando son ocupados por personas de género masculino, mientras que la producción por el género femenino, por lo que resulta interesante estudiar con mayor profundidad las razones y consecuencias de esta singularidad.

En la perspectiva socioeconómica, las BPL se relacionan con dos DHLF: condiciones satisfactorias de trabajo y derecho de género. Dentro de las primeras, las prácticas laborales se enfocan en la salud y bienestar físico, y la conciliación trabajo-familia, mientras dentro de las segundas, las prácticas se enfocan en el apoyo a la salud reproductiva.

Para la conservación de salud y bienestar físico, unas prácticas preparan a los empleados para la actividad laboral con rutinas de calentamiento; otras previenen el cansancio con rutinas de motricidad y mejora continua en la comodidad física del espacio de trabajo; otras más fomentan la capacitación especializada sobre enfermedades y riesgos laborales, así como promueven la valoración física y el permanente seguimiento de la salud. Para la conciliación trabajo-familia, independientemente del género, las prácticas buscan mejorar la integración laboral y familiar de los trabajadores a través de la organización de actividades recreativas, sociales y deportivas.

Para apoyar la salud reproductiva, existen prácticas específicas durante los periodos de embarazo y lactancia. En el embarazo, fomentan el acceso de las trabajadoras a un seguimiento médico, reasignación de tareas, flexibilidad de horarios y de permisos, reducción de riesgos y mejora en la comodidad física del área laboral. Durante la lactancia, la disposición de un espacio específico para ello.

Dentro de la perspectiva filantrópica, se identificaron las prácticas enfocadas al crecimiento personal de los empleados. En esa perspectiva, se reconocieron prácticas laborales que muestran interés por alfabetizar o formar a los empleados ante un eventual crecimiento o desarrollo profesional dentro de la empresa.

Finalmente, en la perspectiva moderna, las prácticas laborales se adscriben a códigos, de ética o de conducta. Los primeros tratan de una declaración general de principios que guían la toma de decisiones entre los miembros de la empresa sobre lo que está bien o mal, mientras que los segundos son más específicos, trazan pautas y procedimientos para determinar si se han producido faltas y las relaciona con consecuencias o sanciones. Estos códigos son usados de manera interna en las empresas. Sin embargo, este tipo de instrumentos deben de ser públicos, visibles y entendibles por todos los grupos de interés (Fair Labor Association [FLA], 2012). En un solo caso se observó su extensión 
hacia los proveedores, pero sin sanciones económicas específicas en caso de incumplimiento.

\section{Conclusiones}

El presente trabajo es solo el inicio de una propuesta de análisis y de discusión en torno a las prácticas laborales de las empresas familiares, considerando diferentes niveles de involucramiento y entornos altamente competitivos. Ha pretendido facilitar la identificación de las prácticas laborales que relacionan a la EF con sus empleados. Durante su elaboración, se pudo constatar la escasa literatura existente al respecto, la confusión de los términos, así como el interés creciente que despierta el tema. Aunado a lo anterior, las características del entorno también parecen ser significativas como factor contingente en el comportamiento de las EF.

El modelo bidimensional de Quazi y O’Brien (2000), cuyo fundamento es una lógica visión-costo para clasificar la RSE sobresale por su pertinencia en el contexto empresarial. En este trabajo, parece haber facilitado la distinción de las prácticas laborales al permitir clasificar los diferentes niveles de involucramiento. Es decir, desde el marco legal vigente hasta la RSE, pasando por las BPL, hasta el reconocimiento de la filantropía.

La información que se pretendió presentar nos conduce a confirmar que las EF no dan evidencia clara de propensión hacia prácticas de RSE (Déniz y Cabrera, 2005; Suárez et al., 2011; CuadradoBallesteros et al., 2015; Cabeza-García et al., 2017), contrario a lo que establece gran parte de la literatura especializada al respecto (Adams et al., 1996; Graafland, 2002; Gallo, 2004; Dyer y Wethen, 2006; Campopiano y De Massis, 2015; Castejón y López, 2016), aunque las prácticas laborales identificadas coincidan con la existencia de relaciones personales, el contacto directo o cierta consideración hacia los empleados (Flören et al., 1993; Uhlaner et al., 2004; Bingham et al., 2011; Neubaum et al., 2012); así como con la promoción de comportamientos éticos entre sus colaboradores (Adams et al., 1996).

Desde una perspectiva de RSE, las prácticas laborales identificadas no parecen ser resultado de la naturaleza familiar de las empresas, sino contingentes a otros factores (Suárez et al., 2011; Déniz y Cabrera, 2005; Cabrera et al., 2011). En este caso, el factor contingente es externo y se relaciona con la presión competitiva del sector y de la globalización. Lo anterior queda evidenciado por la importancia de las auditorias internacionales que valoran aspectos competitivos, sobre la profesionalización operativa de las empresas (ver Tabla 3). Parece que, en las empresas familiares, la iniciativa voluntaria para integrar BPL o RSE en las prácticas laborales es menos significativa que las condiciones comerciales de los mercados internacionales. Las prácticas laborales de las empresas familiares del estudio se destacan por limitarse al marco legal.

Las prácticas laborales (tanto BPL y como prácticas de RSE) parecen poner en evidencia la dificultad que enfrentan las EF del sector para atender las necesidades de sus colaboradores, pudiendo dar indicios de discrecionalidad. También ponen en evidencia la dificultad de un mayor involucramiento voluntario -a través de prácticas de RSE- en aspectos sociales y económicos en relación con las necesidades de sus trabajadores.

Ante esta situación el Estado, a través del robustecimiento de su legislación laboral, puede contribuir en la mejora de las condiciones de los trabajadores; particularmente, cuando el ámbito de operación de la empresa se orienta al mercado interno. Esta situación no sería exclusiva al país de estudio, sino compartida en los países en vías de desarrollo, principalmente los latinoamericanos.

\section{Limitaciones del estudio y futuras líneas de investigación}

Entre las principales limitaciones del presente estudio, se destaca la concerniente al levantamiento de la información y el tamaño de la muestra. Aunque todos los entrevistados fueron los responsables del área de recursos humanos de cada empresa, resulta conveniente cruzar la información obtenida con los empleados y otros grupos de interés directamente relacionados con las empresas ( $v . g r$. cliente y proveedores). Sin embargo, se debe reconocer que, en el caso de los trabajadores, el acceso a esta información puede ser difícil, dado que ellos pueden percibir o experimentar alguna sensación o situación de vulnerabilidad o intimidación. Aunado a lo anterior, también se recomienda 
considerar muestras más grandes y la inclusión de datos cuantitativos. Por ejemplo, incluir encuestas de clima laboral que permitan captar las opiniones del personal que labora dentro de la empresa.

Asimismo, es pertinente incluir otros sectores y tamaños de empresas, dentro del mismo sector moda, con la finalidad de mejorar la comprensión del tema en países en vías de desarrollo y sectores productivos que, cada día más, interactúan de forma dinámica en el terreno global. Con las limitaciones señaladas, sin embargo, se enfatiza aún más la relevancia de continuar con el desarrollo de una investigación cualitativa y cuantitativa sobre las prácticas laborales en EF desde la perspectiva de la RSE, en entornos altamente competitivos, como los que plantean los diferentes países latinoamericanos o las secuelas de profundas crisis económicas (como luego del Covid-19)

\section{Referencias}

Adams, J. S., Taschian, A. y Shore, T. H. (1996). Ethics in family and non-family owned firms: An Exploratory Study. Family Business Review, 9(2), 157-170. doi: 10.1111/j.1741-6248.1996.00157.x

Alto Comisionado de las Naciones Unidas para los Derecho Humanos (ACNUDH). (1996-2020). ¿Qué son los derechos humanos? Recuperado de https://www.ohchr.org/SP/Issues/Pages/WhatareHumanRights.aspx

Amann, B., Jaussaud, J. y Martínez, I. (2012). Corporate Social Responsibility in Japan: Family and Non-Family Business Differences and Determinants. Asian Business \& Management, 11(3), 329-345. doi: 10.1057/ abm.2012.6

Amato, L. H. y Amato, C. H. (2007). The Effects of Firm Size and Industry on Corporate Giving. Journal of Business Ethics. 72(3), 229-241. doi: 10.1007/s10551-0069167-5

Anderson, R. y Reeb, D. (2003). Founding-Family Ownership and Firm Performance: Evidence from the S\&P 500. The Journal of Finance, 58(3), 1301-1328. doi: 10.1111/1540-6261.00567

Astrachan, J. H. y Shanker, M. C. (2003). Family businesses' contribution to the us economy: A closer look. Family Business Review, 16(3), 211-219. doi: 10.1177/08944865030160030601

Benavides, C., Guzmán, C. y Quintana, C. (2011). Evolución de la literatura sobre empresa familiar como disciplina científica. Cuadernos de Economía y Di- rección de la Empresa, 14(2), 78-90. doi: 10.1016/j. cede.2011.02.004

Bingham, J. B., Dyer, W. G., Smith, I. y Adams, G. L. (2011). A Stakeholder Identity Orientation Approach to Corporate Social Performance in Family Firms. Journal of Business Ethics, 99(4), 565-585. doi: 10.1007/s10551010-0669-9

Cabeza-García, L., Sacristán-Navarro, M. y Gómez-Ansón, S. (2017). Family involvement and corporate social responsibility disclosure. Journal of Family Business Strategy, 8(2), 109-122. doi: 10.1016/j.jfbs.2017.04.002

Cairncross, F. (1992). Costing the earth. Boston, MA: Harvard Business School Press.

Campopiano, G. y De Massis, A. (2015). Corporate Social Responsibility Reporting: A Content Analysis in Family and Non-Family Firms. Journal of Business Ethics, 129(3), 511-534. doi: 10.1007/s10551-014-2174-Z

Campopiano, G., De Massis, A. y Cassia, L. (2012). Corporate Social Responsibility in Family Versus Non-Family Enterprises: An Exploratory Study. En A. Lundström, Y. von Friedrichs y E. Sundin (Eds.), Social Entrepreneurship. International Studies in Entrepreneurship, Vol. 29 (pp. 113-154), Springer, Cham. doi: 10.1007/978-3-319-01396-1_6

Castejón, P. J. M. y López, B. A. (2016). Corporate social responsibility in family smes: A comparative study. European Journal of Family Business, 6(1), 21-31. doi: 10.1016/j.ejfb.2016.05.002.

Comisión Nacional de los Derechos Humano [CNDH]. (2016). Derecho humano al trabajo y derechos humanos en el trabajo. Ciudad de México: Comisión Nacional de los Derecho Humanos. México.

Comité de Derechos Económicos, Sociales y Culturales. (2005). El derecho al trabajo (Observación General N. ${ }^{\circ}$ 18). Ginebra: Organización de las Naciones Unidas. Recuperado de http://tbinternet. ohchr.org/_layouts/ treatybodyexternal/Download.aspx?symbolno=E\% 2fC. $12 \% 2 \mathrm{fGC} \%$ - 2f18\&Lang=en

Cromie, S., Dunn, B., Sproull, A. y Chalmers, D. (2001). Small firms with a family focus in the Scottish Highlands and Islands, The Irish Journal of Management, 22(2), 45-66.

Cuadrado-Ballesteros, B., Rodríguez-Ariza, L. y García-Sánchez, I. M. (2015). The role of independent directors at family firms in relation to corporate social responsibility disclosures. International Business Review, 24(5), 890-901. doi: 10.1016/j.ibusrev.2015.04.002

De Massis, A. y Kotlar, J. (2014). The case study method in family business research: guidelines for qualitative scholarship. Journal of Family Business Strategy, 5(1), 15-29. doi: 10.1016/j.jfbs.2014.01.007 
Déniz, M. C. y Cabrera, M. K. (2005). Corporate Social Responsibility and Family Business in Spain. Journal of Business Ethics, 56(1), 27-41. doi: 10.1007/s10551004-3237-3

Diario Oficial de la Federación (DOF). (2009). Acuerdo por el que se establece la estratificación de las Micro, Pequeñas y Medianas Empresas. Extraído de: https://www.economia.gob.mx/files/marco_normativo/A539.pdf

Diario Oficial de la Federación (DOF). (1917). Constitución Política de los Estado Unidos Mexicanos. Texto vigente, última reforma publicada DoF 10-02-2014. Diario Oficial de la Federación. Recuperado de http:// www.diputados.gob.mx/LeyesBiblio/transp/pdf/ CPEUM_150816.pdf

Diario Oficial de la Federación (DOF). (1963). Ley Federal de los Trabajadores al Servicio del Estado, Reglamentaria del Apartado B) del Artículo 123 Constitucional. Texto vigente, última reforma publicada Dof 01-05-2019. Recuperado de Diario oficial de la Federación: http://www. diputados.gob.mx/LeyesBiblio/pdf/111_010519.pdf

Diario Oficial de la Federación (DOF). (1970). Ley Federal del Trabajo. Última reforma publicada DoF 02-07-2019. Obtenido de Diario Oficial de la Federación: https://www.gob.mx/cms/uploads/attachment/ file/156203/1044_Ley_Federal_del_Trabajo.pdf

Dyer, W. G. (1986). Cultural Change in Family Firms: Understanding and Managing Business and Family Transitions. San Francisco: Jossey-Bass.

Dyer, W. G. y Whetten, D. (2006). Family Firms and Social Responsibility: Preliminary Evidence from the S\&P 500. Entrepreneurship Theory and Practice, 30(6), 785802. doi: 10.1111/j.1540-6520.2006.00151.x

Eróstegui, R. (2011). Las buenas prácticas laborales en un contexto de cambio. La Paz, Bolivia: Labor.

FLA. (2012). Fair labor association. Recuperado el 23 de octubre de 2019, de https://www.fairlabor.org

Fletcher, D., De Massis, A. y Nordqvist, M. (2015). Qualitative Research Practices and Family Business Scholarship: A Review and Future Research Agenda. Journal of Family Business Strategy, 7(1), 8-25. doi:10.1016/j. jfbs.2015.08.001

Flören, R. H., Leach, P. y Wijers, E. J. (1993). Handboek van het familiebedrijf. Walgemoed Accountants \& Adviseurs.

Gallo, M. A. (2004). The Family Business and its Social Responsibilities. Family Business Review, 17(2), 135148. doi: 10.1111/j.1741-6248.2004.00009.x

Gavana, G., Gottardo, P. y Moisello, A. M. (2017). Earnings Management and csR Disclosure. Family vs. Non-Fa- mily Firms. Sustainability, 9(12), 2327. doi: 10.3390/ su9122327

Gersick, K. E., Davis, J. A., Hampton, M. M. y Lansberg, I. (1997). Generation to generation: Life cycles of the family business. Harvard Business Press.

Ghauri, P. y Grønhaug, K. (2020). Research Methods in Busi-

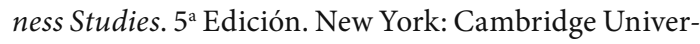
sity Press. doi: https://doi.org/10.1017/9781108762427

Gil, M. A., Jiménez, J. B. y Lorente, J. C. (2001). An analysis of environmental management, organizational context and performance of Spanish hotels. Omega. 29(6), 457-471. doi: 10.1016/s0305-0483(01)00033-0

Graafland, J. J. (2002). Corporate Social Responsibility and Family Business. Artículo presentado en la $13^{\text {ra }}$ conferencia anual del Research Forum of the Family Business Network, Helsinki, Finland.

Gummesson, E. (2015). Innovative Case Study Research in Business and Management. London: Sage Publications.

Handler, W. (1989). Methodological Issues and Considerations in Studying Family Business. Family Business Review, 2(3), 257-276. doi: 10.1111/j.1741-6248.1989.00257.x

Harms, H. (2014). Review of Family Business Definitions: Cluster Approach and Implications of Heterogeneous Application for Family Business Research. International Journal of Financial Studies, 2(3), 280-314. doi: $10.3390 /$ ijfs 2030280

Hernández-Calzada, M. A., Mendoza-Mohen, J., Salazar-Hernández, B. C. (2017). La responsabilidad social en la empresa familiar. Holos, 5, 174-185. doi. 10.15628/ holos.2017.4805

Hispanic in Philantropy (HIP). (2018). Acciones encaminadas hacia buenas prácticas en materia de derechos humanos laborales fundamentales. Sector de la moda en México. Informe final de resultados. Ciudad de México: Hispanic in Philantropy.

Huang, Y. C., Ding, H. B. y Kao, M. R. (2009). Salient stakeholder voices: Family business and green innovation adoption. Journal of Management \& Organization, 15(3), 309-326. doi: 10.1017/s1833367200002649

Instituto Nacional de Estadística y Geografía (Inegi), (2020). Conociendo a la industria textil y de la confección. Recuperado de http://internet.contenidos. inegi.org.mx/contenidos/Productos/prod_serv/ contenidos/espanol/bvinegi/productos/nueva_estruc/702825195649.pdf Javadi, M., y Zarea, K. (2016). Understanding thematic analysis and its pitfall. Demo, 1(1), 33-39. doi: 10.15412/j.jcc.02010107 
Judge, W. Q. y Douglas, T. J. (1998). Performance Implications of Incorporating Natural Environmental Issues into the Strategic Planning Process: An Empirical Assessment. Journal of Management Studies, 35(2), 241262. doi: 10.1111/1467-6486.00092

King, N. y Brooks, J. (2018). Thematic Analysis in Organisational Research. En C. Cassell, A. L. Cunliffe y G. Grandy, The sage Handbook of Qualitative Business and Management Research Methods (pp. 219-236). London: SAGE Publications Ltd. doi: https://doi. org/10.4135/9781526430236.n14

Lagos, D., Betancourt, J. y Gómez, G. (2018). Relación entre gobierno corporativo, control familiar y desempeño en empresas colombianas. Innovar, 28(69), 85-98. doi: 10.15446/innovar.v28n69.71698

Leppäaho, T., Plakoyiannaki, E. y Dimitratos, P. (2016). The Case Study in Family Business: An Analysis of Current Research Practices and Recommendations. Family Business Review, 29(2), 159-173. doi: $10.1177 / 0894486515614157$

Malterud, K., Volkert, S. y Guassora, A. (2015). Sample Size in Qualitative Interview Studies: Guided by Information Power. Qualitative Health Research, 1-8. doi:10.1177/1049732315617444

Marques, P., Presas, P. y Simon, A. (2014). The Heterogeneity of Family Firms in CSR Engagement: The Role of Values. Family Business Review, 27(3), 206-227. doi: $10.1177 / 0894486514539004$

Molina, P. A., Botero, S. y Montoya, J. N. (2016). Empresas de familia: conceptos y modelos para su análisis. Pensamiento \& Gestión, (41), 116-149. doi: https://doi. org/10.14482/pege.41.9704

Muñoz, N. A. (2011). El estudio exploratorio. Mi aproximación al mundo de la investigación cualitativa. Investigación y Educación en Enfermería, 29(3), 492-499.

Neubaum, D. O., Dibrell, C. y Craig, J. B. (2012). Balancing natural environmental concerns of internal and external stakeholders in family and non-family businesses. Journal of Family Business Strategy, 3(1), 28-37. doi: 10.1016/j.jfbs.2012.01.003

Niehm, L., Swinney, J. y Miller, N. (2008). Community Social Responsibility and Its Consequences for Family Business Performance. Journal of Small Business Management, 46, 331-350. doi: 10.1111/j.1540627x.2008.00247.x

Nisim, S. y Benjamin, O. (2008). Power and Size of Firms as Reflected in Cleaning Subcontractors' Practices of Social Responsibility. Journal of Business Ethics, 83(4), 673-683. doi: 10.1007/s10551-007-9647-2
Organización Internacional del Trabajo (оIт). (1930). C029 -Convenio sobre el trabajo forzoso (núm. 29). Recuperado de Normlex Information System on International Labour Standards: https://www.ilo.org/dyn/normlex/ es/f?p=NORMLEXPUB:12100:0::NO:12100:P12100_ INSTRUMENT_ID:312174:NO

Organización Internacional del Trabajo (оIт). (1948). C087 -Convenio sobre la libertad sindical y la protección del derecho de sindicación (núm. 87). Recuperado de Normlex Information System on International Labour Standards: https://www.ilo.org/dyn/normlex/ es/f?p=NORMLEXPUB:12100:0::NO::P12100_ILO_ CODE:C087

Organización Internacional del Trabajo (оIт). (1949). C098 -Convenio sobre el derecho de sindicación y de negociación colectiva (núm. 98). Recuperado de Normlex Information System on International Labour Standards: https://www.ilo.org/dyn/normlex/es/f?p=NORMLEXPUB:12100:0::NO:12100:P12100_INSTRUMENT_ ID:312243:NO

Organización Internacional del Trabajo (OIT). (1951). C100 -Convenio sobre igualdad de remuneración (núm. 100). Recuperado de Normlex Information System on International Labour Standards: https://www.ilo. org/dyn/normlex/es/f?p=NORMLEXPUB:12100:0::NO:12100:P12100_INSTRUMENT_ID:312245:NO

Organización Internacional del Trabajo (oIT). (1957). C105 -Convenio sobre la abolición del trabajo forzoso (núm. 105). Recuperado de Normlex Information System on International Labour Standards: https://www.ilo. org/dyn/normlex/es/f?p=NORMLEXPUB:12100:0::NO:12100:P12100_INSTRUMENT_ID:312250:NO

Organización Internacional del Trabajo (OIT). (1958). C111 -Convenio sobre la discriminación (empleo y ocupación) (núm. 111). Recuperado de Normlex Information System on International Labour Standards: https://www.ilo.org/dyn/normlex/es/f?p=NORMLEXPUB:12100:0::NO:12100:P12100_INSTRUMENT_ ID:312256:NO

Organización Internacional del Trabajo (oIT). (1973). C138 -Convenio sobre la edad mínima (núm. 138). Recuperado de Normlex Information System on International Labour Standars: https://www.ilo.org/dyn/normlex/ es/f?p=NORMLEXPUB:12100:0::NO:12100:P12100_ INSTRUMENT_ID:312283:NO

Organización Internacional del Trabajo (OIT). (1996-2020). Organización Internacional del Trabajo. Convenios y Recomendaciones. Recuperado de https:/www.ilo.org/ global/standards/introduction-to-international-labour-standards/conventions-and-recommendations/ lang--es/index.htm 
Organización Internacional del Trabajo (OIT). (1999). C182 -Convenio sobre las peores formas de trabajo infantil (núm. 182). Recuperado de Normlex Information System on International Labour Standars: https://www.ilo. org/dyn/normlex/es/f?p=NORMLEXPUB:12100:0::NO:12100:P12100_INSTRUMENT_ID:312327:NO

Organización Internacional del Trabajo (OIT) (2005). Recomendación 195 sobre el desarrollo de los recursos humanos, educación, formación y aprendizaje permanente. Adoptada por la Conferencia Internacional del Trabajo, 90ª Reunión, Ginebra.

Organización Internacional del Trabajo (OIT). (2009). Conocer los Derechos Fundamentales en el Trabajo. Recuperado de https://www.ilo.org/wcmsp5/groups/ public/@americas/@ro-lima/@sro-san_jose/documents/publication/wcms_180458.pdf

Organización Internacional del Trabajo (огт). (2017). Informe IV. Principios y derechos fundamentales en el trabajo: Retos y oportunidades. Conferencia Internacional del Trabajo, 106a reunión. Recuperado de Organización https://www.ilo.org/wcmsp5/groups/public/--ed_norm/---relconf/documents/meetingdocument/ wcms_549969.pdf

Perrini, F., Russo, A. y Tencati, A. (2007). CsR Strategies of smes and Large Firms. Evidency from Italy. Journal of Business Ethics. 74, 285-300. doi: 10.1007/ s10551-006-9235-x

Quazi, A., O'Brien, D. (2000). An empirical test of a cross-national model of corporate social responsibility. Journal of Business Ethics, 25(1), 33-51. doi: https:// doi.org/10.1023/A:1006305111122

Quintana, J. (2005). Responsabilidad social en las empresas familiares. Madrid: Foretica.

Reyno, M. (2006). Responsabilidad social empresarial (RSE) como ventaja competitiva (Tesis de maestría).Universidad Técnica Federico Santa María, Valparaíso.

Rueda-Catry, M., Ruiz, V. y Luz, M. (2005). Buenas prácticas de relaciones laborales en las Américas. (No. 993848443402676). International Labour Organization.

Sharma, P. (2004). An Overview of the Field of Family Business Studies: Current Status and Directions for the Future. Family Business Review, 17(1), 1-36. doi: https://doi.org/10.1111/j.1741-6248.2004.00001.x

Sharma, P., Chrisman, J. J. y Chua, J. H. (1997). Strategic management of the family business: Past research and future challenges. Family Business Review, 10(1), 1-35. doi: 10.1111/j.1741-6248.1997.00001.x

Sharma, P. y Srinivas Rao, A. (2000). Successor Attributes in Indian and Canadian Family Firms: A Comparative Study. Family Business Review, 13(4), 313-330. doi: 10.1111/j.1741-6248.2000.00313.x
Stake, R. E. (1995). The art of case study research. California: Sage Publications.

Suárez, M. K. C., Déniz, M. D. L. C. D. y Santana, J. D. M. (2011). Consejos de administración y potencial para la responsabilidad social de las empresas familiares no cotizadas españolas. Investigaciones Europeas de Dirección y Economía de la Empresa, 17(3), 47-67. doi: https://doi.org/10.1016/S1135-2523(12)60120-8

Uhlaner, L. M., Van Goor-Balk, H. y Masurel, E. (2004). Family business and corporate social responsibility in a sample of Dutch firms. Journal of Small Business and Enterprise Development, 11(2), 186-194. doi: $10.1108 / 14626000410537128$

Van Gils, A., Dibrell, C., Neubaum, D. O. y Craig, J. B. (2014). Social issues in the Family Enterprise. Family Business Review, 27(3), 93-205. doi: 10.1177/0894486514542398

Yin, R. K. (2017). Case study research and applications. Design and methods. Los Angeles: Sage Publications.

Yu, A., Lumpkin, G. T., Sorenson, R. L. y Brigham, K. H. (2012). The Landscape of Family Business Outcomes: A Summary and Numerical Taxonomy of Dependent Variables. Family Business Review, 25(1), 33-57. doi: $10.1177 / 0894486511430329$ 